BULLETIN OF THE

AMERICAN MATHEMATICAL SOCIETY

Volume 78, Number 3, May 1972

\title{
CONSTRUCTING ISOTOPIES IN NONCOMPACT 3-MANIFOLDS ${ }^{1}$
}

\author{
BY MARIANNE BROWN
}

Communicated by Steve Armentrout, October 22, 1971

Introduction. Let $M$ be a noncompact, orientable 3-manifold with a (possibly empty) boundary $\partial M$. Suppose $g$ and $h$ are homeomorphisms of $M$ onto itself. When is $g$ isotopic to $h$ ? This question was essentially answered in the compact case by Waldhausen in [3]; roughly the answer given was-when $g$ is homotopic to $h$. We will show that essentially the same answer can be given for a large and interesting class of noncompact manifolds; these manifolds include Whitehead-type contractible open subsets of $R^{3}$. Full proofs of the theorems stated below will be given elsewhere.

I would like to thank Dennis Sullivan, my thesis advisor, for bringing several ideas into sharp focus and for suggesting ways of simplifying some arguments. Thanks are also due David Stone for his generous help in several conversations. For my husband, Edward Brown, who suggested the problem, had innumerable helpful conversations with me, and gave me tremendous amounts of emotional encouragement and support, there are no appropriate words to express my thanks.

Preliminaries. The ambient manifolds considered here are orientable, triangulable and 3-dimensional. By a surface in $M$, we mean a 2-dimensional, triangulable manifold which is properly imbedded in $M$. (Everything is considered from the piecewise linear point of view.) $M$ is an irreducible manifold if every 2 -sphere in $M$ bounds a ball in $M$. For noncompact manifolds this implies that $M$ is aspherical. A surface $F$ in $M$ or $\partial M$ different from a 2-sphere is incompressible in $M$ if $\pi_{1}(F) \rightarrow \pi_{1}(M)$ is a monomorphism. $M$ is boundary-irreducible if each component of $\partial M$ is an incompressible surface. Finally we need the notion of a hierarchy for a manifold. The triple $\left(F_{j}, U\left(F_{j}\right), M_{j}\right), j=1,2, \ldots$, is a hierarchy for $M=M_{1}$ if each $F_{j}$ is a compact incompressible orientable surface in $M_{j}, M_{j+1}$ $=\operatorname{cl}\left(M_{j}-U\left(F_{j}\right)\right)$, where $U\left(F_{j}\right)$ is a regular neighborhood of $F_{j}$ in $M_{j}$ [4], and $M-\bigcup_{j} U\left(F_{j}\right)$ is a collection of balls. If $M$ is compact we require the sequence $F_{j}$ to be finite. For $M$ compact these surfaces have been constructed by Haken when $M$ is irreducible and has an incompressible surface. Waldhausen uses the hierarchy to prove the isotopy theorem in the compact case.

AMS 1970 subject classifications. Primary 58D05; Secondary 55A99, 57C99.

${ }^{1}$ This research is contained in the author's doctoral dissertation submitted to M.I.T. and was supported by a fellowship from the Radcliffe Institute.

Copyright (C) American Mathematical Society 1972 
Noncompact manifolds admitting a hierarchy are called end-irreducible; they have been introduced by E. M. Brown, for manifolds of any dimension using his notions of proper fundamental groups. It can be seen from [1] that the name end-irreducible is appropriate in that it generalizes the idea of boundary-irreducible to ends of a manifold. Moreover any irreducible manifold which is obtained from a compact 3-manifold by removing some incompressible boundary components is an end-irreducible manifold.

Results for irreducible, end-irreducible manifolds. If the manifold $M$ has vacuous boundary, our result asserts that any orientation-preserving homeomorphism of an irreducible and end-irreducible manifold which is homotopic to the identity homeomorphism is isotopic to the identity. Notice that since $M$ is aspherical we are saying that any orientation-preserving homeomorphism which induces the "identity" map on $\pi_{1}(M)$ is isotopic to the identity homeomorphism. More precisely we prove the following two isotopy theorems.

THEOREM 1. Let $M$ be an irreducible, end-irreducible manifold and $H:(M \times I, \partial M \times I) \rightarrow(M, \partial M)$ be a homotopy of an orientation-preserving homeomorphism $h$ to the identity. Then $h$ is isotopic to the identity. [If $H \mid \partial M \times I$ is already the constant homotopy, the isotopy of $h$ to the identity may he chosen fixed on $\partial M$.]

THEOREM 2. Let $M$ be an irreducible, end-irreducible, and boundaryirreducible manifold. Assume $\partial M \neq \varnothing$. Suppose $H: M \times I \rightarrow M$ is a homotopy of an orientation-preserving homeomorphism $h$ to the identity and suppose that $H$ is a proper map when restricted to each component of $\partial M \times I$. Then $h$ is isotopic to the identity.

(By a proper map we mean that the inverse image of compact sets are compact.) In the above theorems we only use the fact that $h$ is orientationpreserving for manifolds that

(a) are bundles with fiber $R$ over closed surfaces, (in Theorem 1),

(b) are bundles with fiber $I$ over open surfaces, (in Theorem 2) or

(c) when some component of $\partial M$ is a plane or an open annulus, (in Theorem 1).

To see that in Theorem 2 it is necessary to assume $H$ is a proper map when restricted to components of $\partial M \times I$ we look at the following example. Let $M$ be a solid torus with two disjoint longitudinal curves removed from $\partial M$. Then $\partial M$ consists of two open annuli. Let $h$ be the homeomorphism of $M$ which rotates the torus so that the boundary components are interchanged. Then $h$ is homotopic to the identity but not isotopic to the identity.

Other examples of end-irreducible manifolds will be given in the next section. 
An outline of the proof of Theorem 1 for a manifold $M$ with vacuous boundary. In [1], it is shown that $M$ has an exhausting sequence $\left\{C_{n}\right\}$ of submanifolds with the following properties:

(1) $C_{n}$ is a compact, connected manifold,

(2) $C_{n} \subset C_{n+1}$ and $\bigcup_{n} C_{n}=M$,

(3) components of $\partial C_{n}$ are incompressible.

Now one can show that $\left\{C_{n}\right\}$ may be chosen so that

(4) $H\left(C_{n} \times I\right) \subset \dot{C}_{n+1}$.

We show by an inductive procedure that if $h \mid C_{n}$ is the identity and $H \mid C_{n} \times I$ is the constant homotopy, then there is an isotopy of $h$ fixed on $C_{n}$ so that the new homeomorphism (which we will still call $h$ ) is the identity on $C_{n+1}$. Moreover we show that one can change the homotopy to be constant on $C_{n+1}$. Let $F_{1}, \ldots, F_{k}$ be the components of $\partial C_{n+1}$. We first show that since $F_{1}$ and $h\left(F_{1}\right)$ are incompressible surfaces which are homotopic via $H$ in $C_{n+2}$, there is an isotopy of $M$ fixed on $C_{n}$ which carries $h\left(F_{1}\right)$ onto $F_{1}$. (We use here that if $M$ is a product bundle, then $h$ is assumed orientation-preserving.) Changing $h$ by this isotopy-still call the homeomorphism $h$-we now have $h\left(F_{1}\right)=F_{1}$.

The fact that this isotopy can be chosen fixed on $C_{n}$ rests heavily on the fact that $H \mid C_{n} \times I$ is already constant. Thus we now try to change $H \mid F_{1} \times I$ by a homotopy to the constant homotopy. First we attempt to homotope $H \mid F_{1} \times I$ rel $F_{1} \times \partial I$ to a map into $F_{1}$. We show the only difficulties arise when $M$ is a bundle over a closed, nonorientable manifold. Here we again use the assumption that $h$ is orientation-preserving. Next we show that unless $F_{1}$ is a torus, $H \mid F_{1} \times I$ is homotopic rel $F_{1} \times \partial I$ to the constant homotopy. If $F_{1}$ is a torus it may be necessary to change $h \mid F_{1}$ by an isotopy before one can homotope $H \mid F_{1} \times I$ to the desired state. We continue - by induction on the number of components in $\partial C_{n+1}$ - to change $h$ by an isotopy fixed on $C_{n}$ and to change $H \mid \partial C_{n+1} \times I$ by a homotopy so that in the end, $h \mid \partial C_{n+1}$ is the identity and $H \mid \partial C_{n+1} \times I$ is the constant homotopy.

Since $\operatorname{cl}\left(C_{n+1}-C_{n}\right)$ is a manifold with boundary, it admits a hierarchy $G_{1}, \ldots, G_{r}$ (see [2]). Again we inductively isotope $h$ and homotope $H$ without disturbing our previous changes so that the end result is that $h$ is the identity on all the $G_{i}$. Hence $h$ is the identity on $C_{n+1}$ except for a collection of 3-cells. Using Alexander's Theorem, we conclude $h$ is isotopic to the identity on $C_{n+1}$; using the fact that $M$ is aspherical we have no obstructions to homotoping $H$ to the constant homotopy. This concludes the argument.

Results for irreducible, eventually end-irreducible manifolds. $M$ is eventually end-irreducible if there is a compact subset $C$ of $M$ such that $M-C$ is end-irreducible, or equivalently that $M$ eventually has a hierarchy, 
i.e., $M-C$ has a hierarchy. For the isotopy results for such manifolds the following example shows that it is necessary that we assume the homeomorphism $h$ is proper homotopic to the identity. Let $T_{0}$ be a solid torus linked in the solid torus $T_{1}$. (See Figure below.)

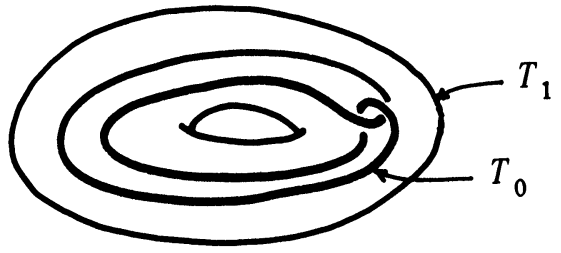

Let $h: R^{3} \rightarrow R^{3}$ be a homeomorphism of $R^{3}$ such that $h\left(T_{0}\right)=T_{1}$. Then $W=\bigcup h^{i}\left(T_{0}\right)$ is the contractible open subspace of $R^{3}$ described by Whitehead in [5]. By results in [6], one can show that $W$ is an eventually end-irreducible manifold. The homeomorphism $h$ maps $W$ onto itself. Moreover since $W$ is contractible $h$ is homotopic to the identity. Again the results in [6] show that $h$ is not proper homotopic to the identity and hence it certainly is not isotopic to the identity.

THEOREM 3. Let $M$ be an irreducible, eventually end-irreducible manifold and $H:(M \times I, \partial M \times I) \rightarrow(M, \partial M)$ a proper homotopy of a homeomorphism $h$ to the identity. Then $h$ is isotopic to the identity. If $H \mid \partial M \times I$ is the constant homotopy, then the isotopy of $h$ to the identity may be chosen fixed on $\partial M$.

\section{BIBLIOGRAPHY}

1. E. M. Brown and T. Tucker, On proper homotopy theory for noncompact manifolds, Trans. Amer. Math. Soc. (to appear).

2. W. Haken, Uber das Homoömorphieproblem der 3-Mannigfaltigkeiten. I, Math. Z. 80 (1962), 89-120. MR 28 \# 3410.

3. R. Waldhausen, On irreducible 3-manifolds which are sufficiently large, Ann. of Math. (2) 87 (1968), 56-88. MR 36 \# 7146.

4. J. H. C. Whitehead, Simplicial spaces, nuclei, and m-groups, Proc. London Math. Soc. 45 (1939), 243-327.

5., A certain open manifold whose group is unity, Quart. J. Math. Oxford 6 (1935), 268-279.

6. J. H. C. Whitehead and M. H. A. Newman, "On the group of a certain linkage," in Mathematical works of J. H. C. Whitehead, Vol. II, Pergamon Press, Oxford and New York, 1963. MR 30 \# 4667b.

Department of Mathematics, Dartmouth College, Hanover, New Hampshire 03755 